\title{
Potential contribution of the Infrared Industry in the future of IoT / IloT
}

\author{
by *Andres E. Rozlosnik \\ * SI Termografía Infrarroja, Sánchez de Bustamante 2144 6B CP 1425 Buenos Aires - Argentina \\ aer@termografia.com
}

\begin{abstract}
Internet of Things and Industrial Internet of Things begin its journey in several areas of our lives. Is a new wave of connectivity that is going beyond, desktop, tablet, smartphones, laptops or notebooks (internet of people). The goal to achieving is that things / objects become beings with a curiosity like humans and interconnected with each other. We do not know now how far the development will go. What we are sure is in this new digital era of hyper-connectivity the Infrared Industry: thermal imaging, InGaAs, thermal sensors, hyperspectral, there is much that can contribute.
\end{abstract}

Key words: Internet of Things (IoT), Industrial Internet of Things (IloT), IP6V, Infrared devices, UAV/Unmanned aerial vehicles-based thermal infrared, Artificial Intelligence

\section{Introduction}

This hyper-automated era will cover our daily life like cities, homes (domotic) and buildings. Also, in fields such as defense and security, retail, agriculture, health, energy generation, transportation, industries, testing, sports, wearables, are going to be part of this new hyper-connected network. In all fields there is surely an infrared application that can be developed. All technical disciplines and human activities could be involved. Looks like a cultural change to come. The potential implications of the loT are much bigger in scope. All technical disciplines and human activities could be involved in interconnected devices. (IP) Some machines could start to replace human decision-making.

What is the input that the infrared industry can provide? Just to remember some basic photonics / photometry concepts: Light is the supreme messenger. Light tells us something about the cosmos, the make-up of our bodies, and nearly everything in between. [NIST Radiometry [1]. On the other hand, objects over $>0$ Kelvin (T) have its own infrared light invisible to human eyes (radiance $\mathrm{L} \mathrm{W} \cdot \mathrm{sr}^{-1} \bullet \mathrm{m}^{-2} /$ radiance intensity I W• $\mathrm{sr}^{-1} /$ radiant exitance $\left(\mathrm{W} \cdot \mathrm{m}^{-2}\right)$. That invisible light (radiance) is one that also can give us a lot of information about what we are looking at.

Non-Contact thermal sensors/detectors measure from objects the radiance flux emitted, reflected, transmitted from passive or active origin. Radiance is related to temperature. Temperature differences generate heat transfer. Radiance also can be affected by convection and conduction heat transfer. Infrared Radiance detected brings knew knowledge of what we are looking at. We can vary that radiance exitance by thermally exciting the object to explore its interior. For opaque bodies (graybody) radiance exitance is proportional to its ability to emits $(\varepsilon=1-\rho)$, a constant and T at the fourth (4) power. Unlike thermal energy which is radiated, SWIR is a reflected energy (not at high temperatures) like visible light. (SWIR sensor-- InGaAs)

Over the years Infrared industry (thermography and much more) challenge, develop hardware /software to transform that infrared radiation (radiance) from a target in a representative visible image with the highest SNR, with the smallest NETD, the highest resolution (number of pixels \& size detector pitch) and accuracy, and so on. When thermal imaging, thermography and thermometry began in the commercial market, let's say more than 40 years from now, the community used to say applications are going to be only limited by the imagination. The results show that it was a correct thought and today is current. Although there are some infrared applications that require more maturity the technology spread everywhere, and its performance, quality and price of hardware /software was improved over the years.

Now we are in a moment in the history of science -technology evolution in which all things, objects, instruments etc. active and passive has the potential to exchange info or instructions (Infrared industry devices included) through the network.

In many areas we can imagine a thermal sensors and thermal imaging cameras (radiometric and non-radiometric) in which the involvement can intervene alone or integrated with other technologies. Some of the equipment developed by the infrared industry at present will very useful in the network but as the development progresses towards in the hyper connectivity, new applications and developments will emerge.

\section{IoT / IloT Conceptualization / Challenges - Risks}

\subsection{Conceptualization}

IoT is equal to a revolution in the relationships between people and objects, even between objects and things directly, which will connect them with each other and with the network and, in turn, will offer data in real time. In other words, it has to do with the digitalization of the physical world. loT means potential smart, connected objects. loT often

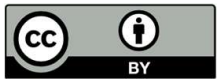


centres on comfort for individual consumers. Instead, IloT aims to improve the efficiency, safety, and productivity of operations with a focus on return on investment. These are very general definitions.

IloT tries to make machines more efficient and easier to control. Factories, heavy industries, mining are possibly sectors that have been working with concepts for much longer, thanks to robotics, SCADA, DCS, etc. The exchange of data for operation, production and maintenance purposes make industrial equipment much more efficient and useful, and creates a safer environment. M2M is a subset of IoT/lloT, which tends to focus very specifically on machine-to-machine communications. M2M is often used interchangeably with the loT.

The above IoT and IloT definitions are too general and do not correspond to strict standardization. It is more a generalization of how the industry sees it. For infrared devices, there will be applications in both loT and IloT. They depend on how they are classified and how they are developed. From now on, for simplification purposes, the term loT will be used in this document in reference to both although in some cases you can see loTT as well. In reality it is the same concept only that one is more on the side of the industrial applications.

\section{2 loT Architecture - Framework - General Challenges - Risks}

The implementation of the Internet of Things (loT) and the Industrial Internet of Things (IloT) presents enormous challenges in different areas, not only in the technical field, but also in security, economy, etc. It is clear that it is not possible to generalize and each application should evaluate the challenges, advantages and risks. The spectrum of application is very broad and, in some cases, could involve high risks of failure with potential catastrophic consequences.

First, we must recognize that the loT / IloT is still in an immature stage, but exponential growth is expected in the near future. It is in a period of very early evolution. Regardless of the final application, what are the key factors for the successful development of IoT / IloT? What would the necessary requirements to have a reliable and secure network to develop an loT - IloT be? What is missing to achieve sustainable development in the new generation of unlimited internet networks?

Let's have a quick look at the development of the general situation, its main factors and concerns for potential developers and end users. In summary, we found eleven (11) basic representative topics for a healthy and secure evolution of IoT / IloT; namely: platforms-standardization, network connections, sensors, embedded processor, cyber security, artificial intelligence, available IP addresses, higher connection speed, investments, health implications and potential impact on employment -taxes (From 2.2.1 to 2.2.11)

\subsubsection{Platforms - Standardization}

Some platforms are just connectivity, while others are integrated solutions that offer connectivity, hardware, software, analytics, user interface and security (platforms layers). loT platforms facilitate and organize interactions between each of these layers, as well as with other systems of an organization / company. Probably there are more than $>500$ platforms developed up today and grow exponentially every year. There are platforms for specialized topics and others that can be used for a variety of applications. On the other hand, it must be considered that, except in very special cases, IoT is not a unique technology (as in this case the infrared sensors). It is a combination of sensors (or peripheral sensors), devices, software, and analytics that work together to obtain and interpret valuable and processable data from the loT. The choice of a suitable platform depending on the case is vital for the success of the (+ cloud) development. Whatever the eligible platform, it would be always convenient to keep it updated. Implementation costs (2.2.9) can be high.

Experts say non-standardization is the main obstacle to rapid and lasting progress. Currently, the biggest problem loT faces is the lack of consistent standards in all layer's platforms, especially in communication protocols.

Without a common communication method, the devices will only be able to speak with their own brands and severely limit the usefulness of the connected machines. For example, if a company that develops smart cameras is different from a company that develops smart home technology, the chances of their products communicating are minimal. That's because different devices often use different communication protocols (LP-WPAN, Bluetooth-BLE, NFC, cellular- network, satellite networks, Wi-Fi, Wi-Fi HaLow, Z-Wave, Zigbee, RFID, SigFox, etc.) resulting in a lack of interoperability and a complicated experience for end users. However, if the two companies (cameras + domestic technology) use the same standard for connectivity, the ability to exchange and use information would be much more likely. If that is not done, there will be interfaces plus interfaces, several types of interfaces. Standards are needed to prevent interfaces from becoming, in some way, market development bottlenecks.

There is a trade-off between power, range, intermittent connectivity, bandwidth, and security issues in the selection of a communication protocol for each application. This is one of the elements that should be considered in the harmonization (standardization) of protocols between brands and developers. It should be noted that not all loT protocols must be or are wirelessly designed. For example, sensor units that are installed within a factory or building automation system can use wired (fixed) network technologies such as Ethernet and not necessarily some of the above communication protocols. An advantageous thing in the future would be platforms that admit different types of connectivity, this flexibility to expand and integrate the business into the future. The communication protocols Wi-Fi HaLow mention above is Wi-Fi low power that works with batteries and can be used for remote sensing and control applications. Devices sleep when not transmitting data and need just few milliseconds to wake up.

As we said there are many platforms $(>500)$ with different functionalities, architecture, and scopes. Just name few popular: Amazon Web Services (AWS), Ayla, Cisco Jasper, Exosite, General Electric (GE), LogMeln, PTC, SAP, Zebra Technologies, Microsoft loT Azure, Google Cloud's loT Platform, IBM Watson lo, Amazon Rekognition, Bosch 
Software Innovations GmbH, AT\&T Home Grown •C3 loT, Xively Platform ... and so on In relation software's used for artificial intelligence $(\mathrm{Al})$, we find that Python and Java are probably the most popular. There are more. Python is an interpreted programming language, oriented to objects and of high level for general purposes. Python is on behalf of the Python Software Foundation.

\subsubsection{Network Connections}

IoT / IloT require very reliable network connections with high availability, coverage and lower cost, which is not available today in many places in the world. (classified as wired or wireless) The Internet of Things (mainly wireless) can be segmented into massive loT applications and critical loT applications. The quality of the connection and how stable it turns out is very important for the development of this type of projects and in many applications that it may be necessary to develop. The large-scale use of different loT-connected devices promises to change many aspects of our daily life and how we relate to each other.

While many loT networks may be unrelated from the public Internet, future LTE-5G mobile service should be an important driver for the loT. Likewise, it seems that in the near future 5G fixed (router) wireless broadband (radio signals rather than cables) access will be able to be installed at homes, so phone companies will be able to provide similar service than ISP.

A concern about the network connection may be the end of the internet neutrality. Although many of the loT developments are expected to be in private networks, those in open networks like small businesses or loT startups could be affected with a higher ISP cost. Whether the end of neutrality will be a fact or not, only time will tell.

\subsubsection{Sensors}

Sensors with affordable prices are important for the development of loT. In general, they are expensive, especially infrared devices. The fall in prices of hardware and sensors is a reality and goes hand in hand with massive use of these devices. Each of these sensors (small sensors otherwise known as intelligent dust) or integrated devices should have their autonomous IP in the network. Sensors /detectors are the source of the data for the realization of loT.

In the future, we can imagine infrared devices networked to another intelligent sensor integrated in loT for a common objective or application. Just to get an idea, the most popular ones can be mentioned in alphabetical order: acceleration, acoustic anemometer (sound pressure), artificial vision (visible image cameras), biometric sensors, current, deformation, displacement, distance, extensometer, force, gas sensors, GPS, gyroscope, humidity, tilt, laser scanners, LiDAR, light intensity, limit sensor switch, linear and angular position, linear and angular speed, location, motion capture sensors, motor controllers, motion sensors, pressure, proximity and presence, telemeters, seismometers, resistance, temperature (contact), torque (deformation), torsion, ultrasound, voltage.

The data obtained from a sensor or sensor system can be combined with data from the cloud, other systems and collected, analyzed and exchanged in new ways. The sensors have the capacity to take measurements such as temperature, air quality, movement and electricity. Depending on the technology and the manufacturer, some sensors can have a degree of memory, which allows them to record and save a certain number of measurements. This can save energy, which is very important in the loT network.

A clear example of sensor integration (cloud involvement) is the development of autonomous cars. They use a variety of sensors to detect their surroundings so that the car can reach its destination. An autonomous car (also known as a driverless car) is a vehicle that can sense its environment and navigating without human input. Sensors like LiDAR, radars, GPS, regular visible cameras, ultrasonic sonars, accelerometers, gyroscopes, thermal cameras and other readouts are fused into a common goal.

Sensors in general are classified according to their energy sources: active versus passive. The active sensors emit their own energy and then perceive the response of the environment the reflected energy. (e.g. Radar) Passive sensors simply receive energy that is produced externally to the sensor. An infrared camera or infrared thermometer is built with a passive sensor. It receives infrared radiation emitted and reflected (radiosity from opaque object) from the target. Hopefully someday could be developed an infrared sensor active -passive (or all in one) for NDT active infrared test.

It is understood that, for each potential IR application (imaging FPA or point sensor) with loT, the type of sensor to choose will depend on the application and, consequently, resolution (radiometric, spectral, spatial, and temporal) should be considered and analyzed.

Many of the sensors of other technologies mentioned above are sensors that act or measure when they are in contact with the object to be measured. Temperature can also be measured by different types of contact sensor, but it is not the scope of this paper

Basically, in the infrared industry, the detectors-sensors could be single element, scanned single element, linear arrays and two-dimensional - focal plane array (FPA-cool /uncooled). The most popular materials and substrates are Si, InGaAs, PbSe, InSb, HgCdTe (MCT) QWIP (Quantum Well), JT detector, vanadium oxide (VOx), amorphous silicon ( $\alpha$ $\mathrm{Si}$ ), and the new SWaP detectors (HOT) : nBn sensors \& SLS (strained layer superlattice) and T2SL (Type II Super Lattice). Probably, vanadium oxide (VOx) and amorphous Silicon ( $\alpha-S i)$ are the ones that will be used more in the future of loT (uncooled technology).

Most of the detectors that we mentioned in the last paragraph are used for infrared imaging applications. (radiometric or non-radiometric) We cannot forget about fixed or portable non-contact infrared thermometers. They are 
also called radiation pyrometers or optical pyrometers (e.g. thermopile detector) and they have a very wide field of application in industry and medicine. They are part of the infrared industry and could be integrated into loT. (Wi- $\mathrm{Fi}$,.. Bluetooth)

\subsubsection{Embedded Processor}

loT welcomes research in embedded systems in order to improve performance sensors and actuators, verification, etc. An embedded system is a computer system designed to perform one or a few very specific functions, usually in a real-time computer system. A sensor with an embedded processor is a programmable computer but, in itself, it is not intended to be a general-purpose computer. An embedded system means a computer inside the product. An embedded processor is a CPU chip used in a system which is not a general-purpose workstation or desktop computer. Concerning power efficiency, many systems are battery-powered and must conserve power to maximize the usable life of the system. The Internet of Things can be considered as a system composed of a large number of autonomous devices within a larger integrated global system connected to the cloud.

The challenges posed are low power consumption and memory capacity. The other important device in the layout of the integrated processor is the actuator. The actuator is a device that converts the received electrical signal into action.

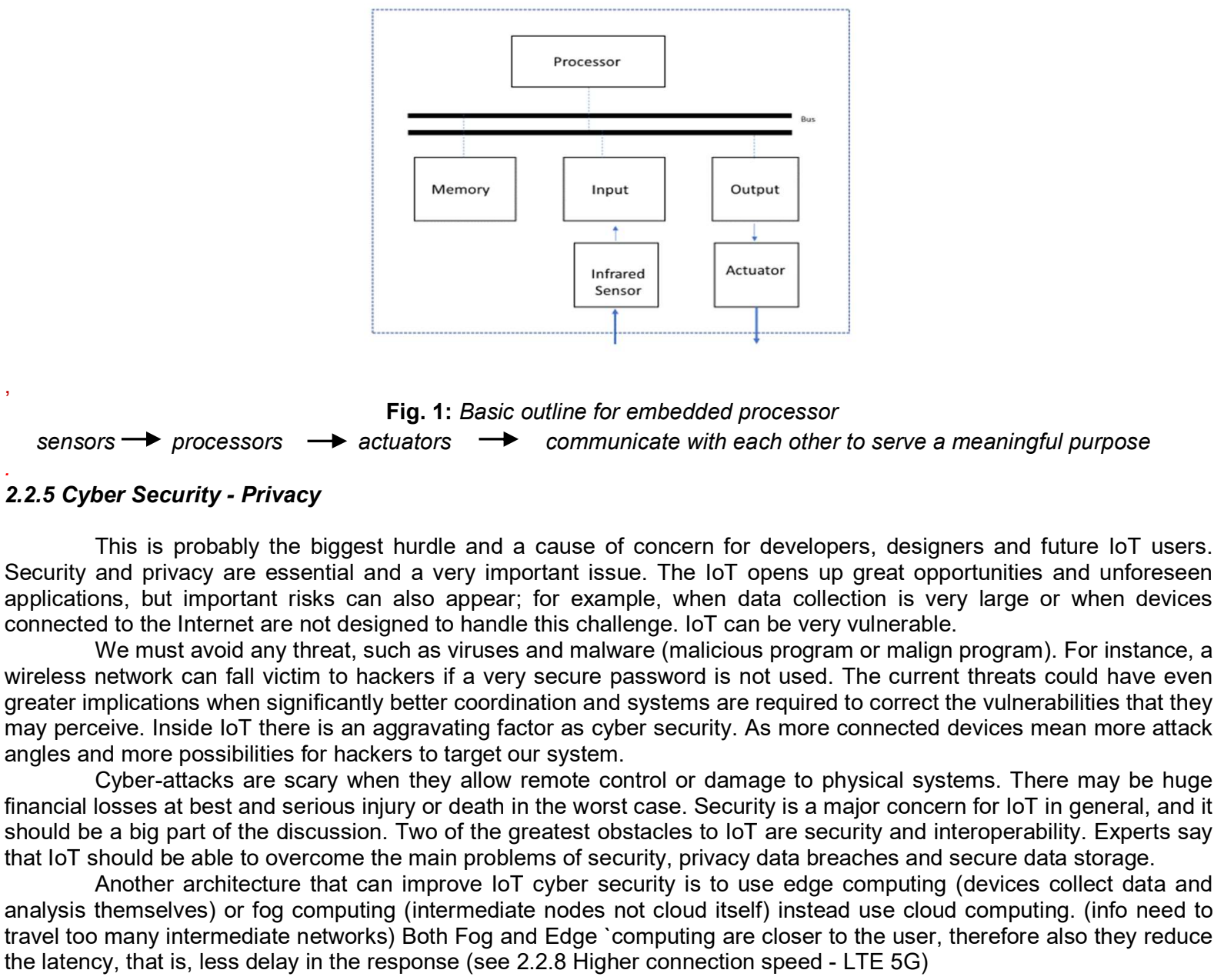

\subsubsection{Artificial Intelligence}

There are two types of intelligence: human intelligence (natural intelligence, $\mathrm{NI}$ ) and artificial intelligence (AI). In artificial intelligence there are different approaches but, there is agreement that artificial intelligence is a subfield of information technology (subfield computer science) whose goal is to develop computers capable of doing some specific things that humans normally do. Commonly, artificial intelligence is classified into two branches: weak artificial intelligence (weak $\mathrm{Al}$ ), which focuses on a narrow task, and artificial general intelligence ( $\mathrm{AGl}=$ strong $\mathrm{Al}$ ), which can successfully perform any intellectual task that a human being can perform. AGI, some called cognitive computing, which simulated 
human thought processes in a like computerized model. The computer can simulate the way the human brain works through pattern recognition and natural language processing. Cognitive computing goes beyond Al and contemplates more processing than Al. Cognitive intelligence in systems tries to achieve the behavior that humans do, reasoning, thinking, and memorizing.

Computers today can surpass man but only in relation to the speed of operations, precision and the storage of information. Computers can do algorithmic operations that solve problems that only have a solution. Computers can think harder and faster than humans. Nevertheless, human intelligence is able to improvise, to explore other facets of reality. The question is what the Al limit is? How far can get? What can happen when machines become smarter than humans? The group of founders of Al and robotics companies want global treaty to ban killer robots by 2019 .

Machine learning-ML (subset $\mathrm{Al}$ ) is the techniques that allow computers to learn, in other words, it is the science of making computers act without being really programmed. ML is a branch of artificial intelligence, which combines algorithms, software and sensors. It is one of the fastest growing areas in the field of technology. It is also able to identify patterns and develop algorithms in large-scale data (Big Data = raw resources of $\mathrm{Al}$ ) then quantify the information to analyze and predict. (e.g. in thermal images) Machine learning systems can improve their performance with the large amount of data available on each topic, which grows day after day. Now there are billions of examples instead of hundreds. The idea is that the more data are fed into a computer involved in machine learning, the smarter it will become. Big data is collected or handling structured or unstructured.

The Al used in thermal cameras will go beyond traditional functions such as monitoring and recording by offering advanced video analysis functions; for instance, comparison of thermal contrast, facial recognition (SWIR), people counting, extraneous movements of people or vehicles, and so on. Advances in artificial intelligence will mark how much more automated and proactive some areas of application of the loT will be.

\subsubsection{Available IP Addresses - IPv6}

This means enough individual IP addresses so that all possible objects and people can connect to the Internet. IPv6 was designed to allow the growth of the Internet in future generations. As mentioned earlier, every device that connects to the Internet uses an IP address that must be unique. Other existing or developing solutions consider "sharing" the same IP address between different devices, which leads to complicated networks that become fragile and at the same time difficult to analyze to correct problems. Just like a phone number helps you communicate with another phone, computers are provided with an IP address so that you can communicate with Internet services, websites, etc. The IP6V protocol began to be implemented from June 6, 2012 and the existing IPv4 will be gradually replaced as it is practically saturated. The explosive growth in mobile devices and wireless portable devices has created the need for additional blocks of IP addresses. IPv6 means mobility. That is, a device can retain a virtual point of presence in one network while it is connected to another. Version 5 (IPv5) was reserved before IPv6, but it was never widely implemented and will not be used publicly. Due to the shortage of IPv4 addresses, much of the Internet is based on NAT (Network Address Translation). IPv6 is much better than IPv4 to ensure that Internet traffic reaches the correct destination without being intercepted.

IPv6 is an important step towards the implementation of IoT. IoT is conceived as a network of billions of interconnected smart devices, a billion people interacting and a potential for millions of online e-businesses. IPv4 currently supports a maximum of approximately 4,300 million unique IP addresses. Meanwhile, both live on the web. Dual stack means transition technologies. According to some forecasts, there will be around 30 billion "things" connected by 2020. Therefore, without IPv6, the progress of loT / IloT is totally limited.

Below is a rough estimate of the number of addresses that each protocol can generate:

- Total IPv4 space: 4,294,967,296 / addresses-- $\left(2^{32}\right)$ - 32 bit ------- Saturated-Exhausted

- $\quad$ Total IPv6 space: 340,282,366,920,938,463,463,374,607,431,768,211,456 / addresses - $\left(2^{128}\right) 128$ bit --approximately $\left(3.4 \times 10^{38}\right)$-- (IPv6 addresses can be created automatically from the local system's MAC address)

\subsubsection{Higher Connection Speed - LTE 5G}

It is also related to 2.2.2 Network Connections. The $5 \mathrm{G}$ represents the fifth generation of wireless technologies and will be faster than $4 \mathrm{G}$ long term evolution (LTE) and continues to introduce new capabilities to better support the loT.

The 5G standard is well-defined, it is a technological evolution of mobile networks that improves the loT (Internet of Things), since its main application will be the objects connected to the web, whether they are autonomous cars, homes, or smart cities with artificial intelligence included applied to industry and health. LTG 5 G networks will greatly increase the number of devices (including infrared sensors) connected directly to each other, making communication more efficient and, therefore, with a lower expenditure of battery power. LTE 5G will make communications become very fast almost in real time, putting mobile internet services on a par with office services. Delivering high speeds, $5 \mathrm{G}$ has the potential to handle many more mobile devices at the same time without network congestion and handle those connections with low latency. It is worth mentioning that the lower the latency, the faster the activated device reacts remotely.

Thus, the three main characteristics of the $5 \mathrm{G}$ are based on the availability of greater bandwidth (with standard speeds of $1 \mathrm{GBPS}$ and peaks of $15 \mathrm{Gbps}$ ), the extreme reliability of the network with very low latency (of 1 millisecond, compared to 100 milliseconds of $4 \mathrm{G}$ ), and the possibility of exponentially increasing the number of connected devices. A movie can be downloaded in less than three-four seconds, cars can automatically detect and save obstacles on the road, and doctors can perform complex surgical procedures using shape robots (telemedicine). Another way to reduce latency is to have a part of the large cloud at a lower distance as we said is called edge computing or fog computing. 
It is likely that $5 \mathrm{G}$ will become the glue that unites many of our critical technologies (including the infrared industry) and it will place mobile telephony and Internet operators in general at the center of modern global civilization in a big way. As it was pointed out in section 2.2.2. Network Connections, the routers of the $5 \mathrm{G}$ networks are in the process of development. On the other hand, today devices such as tablets support both Wi-Fi and Cellular- Data. Experts say that probably in future both communication protocols will compete and perhaps complement each other. . WiMAX is categorized as an LTE technology.

\subsubsection{Applications Investments (platforms) - Investments for Individual Application - High Cost of Implementation}

There is uncertainty as to whether the loT will bring the benefits promised. There are interoperability concerns. One of the big challenges for the industry or the eyes of the investors is how to manage the large volume of loT data (big data) that is going to be produced and to create value out of it. Investments will be made in areas in which investing makes economic sense.

\subsubsection{Health Potential Impact}

Radio-frequency and electro-magnetic radiation (RF-EMF), which are released continuously, have become one of the most toxic forms of pollution and a major health concern. Some questions have arisen. How should we protect ourselves from harmful EMF radiation? What are the effects on human health? May RF-EMF represent a long-term health risk? The mobile phone -like coffee and other possible carcinogens- was categorized as 2B by the International Agency for Research on Cancer (IARC). Cell towers and Wi-Fi networks have been classified as 2B too. Radiation with loT should increase since it should be taken into account that the connections will be extended considerably. Not only people will be connected but the things and most of them will be wireless.

\subsubsection{IoT Automatization Impact on Employment -Taxes}

There are many people who believe that this process of emerging technologies (loT, robotics, drones, etc.) will create a serious employment problem. Apparently, many jobs will be outdated as loT grows, but these new technologies are going to generate many new opportunities. There are going to be new openings. We must become aware of the need to educate and prepare new generations for new roles and technical specialization. Education is the basis of everything and even more in this case. We have to start training new generations because new opportunities in asset management, robotic design and maintenance, remote diagnostics, analytics expertise, imaging technology big data and data analytics will arise and they will be very important for the proliferation of loT. Apart from that, emerging technology could create jobs in areas such as network security, hardware and software development, machine learning, AutoCAD GPS, electronics design, sensors development, programming and algorithm generation, etc. These new jobs or recycling old jobs will cause the need for a highly skilled workforce which will have to be trained.

So as automation accelerates, jobs will be lost, therefore, and less taxes will be paid, in part will be compensated with new jobs and tasks. Now, if these new tasks do not compensate for losses, robots - autonomous networks should they pay taxes? In reality what will surely happen is that the human being will adapt perfectly what will come.

\section{3. loT Infrared Industry Potential Applications - Ground-Based (Robots / Portable / Fixed / Vehicles) - Aerial (Drones)}

Find below Table (Fig. 2) that shows the list of ground-based and aerial devices. Then we expand the applications detailed in the table: sections 3.1 (Ground-Based) and 3.2 (Aerial) Some of the infrared areas or specific applications listed in those sections have years of ongoing in the field with successfully results and without the existence of connectivity and IoT. It is expected that with loT / Al the infrared industry can be integrated into loT many more applications and other types of sensors and cross-platform software. It is a good opportunity to show to those who don't know the technology the broad potential it has. For each specific or general application, a platform (2.2.1 Platforms - Standardization) should be chosen or designed. Surely no loT standard platform satisfy the wide spectrum of uses of infrared sensors / detectors both single or integrated with other sensors from different applications.

\begin{tabular}{|c|c|c|}
\hline \multirow{5}{*}{$\begin{array}{c}\text { Infrared } \\
\text { Potential } \\
\text { Applications } \\
\text { (IoT) }\end{array}$} & \multirow{4}{*}{$\begin{array}{c}\text { Ground-Based } \\
\text { Sensors-Cameras } \\
\text { - Pyrometers } 3.1\end{array}$} & Robots \\
\hline & & Portable \\
\hline & & Fixed-Mount \\
\hline & & Autonomous Vehicles-Machinery \\
\hline & $\begin{array}{c}\text { Aerial Sensor- } \\
\text { Cameras-3.2 } \\
\text { Drones -UAV }\end{array}$ & $\begin{array}{l}\text { Multi-Rotors-Single Rotors- } \\
\text { Fixed-Wing Planes Airborne }\end{array}$ \\
\hline
\end{tabular}

Fig. 2: Outline of applications --- (Note pyrometer is a radiation thermometer) 


\subsection{Ground-Based (Robots / Portable / Fixed / Vehicles)}

So, follow this paragraph the list of fields of applications (in alphabetical order- 3.1.1 TO 3.1.15) for ground-based devices with potential in loT. All of them are already successful in their area of infrared application without being used to a large extent in the loT network. As time goes by and the IoT is strengthened, it will be possible to apply it to most of the same areas, and more, with different degrees of integration into the industry. It seems that the possibilities are endless. The applications with infrared sensors / detectors can be integrated simultaneously in loT with other types of sensors. Keep in mind that loT suggests intelligent automation, but, in fact, in many cases, automation already exists. (e.g.M2M) loT could make the system more efficient and proactive and it will be able to have all the information integrated into the cloud. This comes closely related to real-time communications and data analytics.

\subsubsection{Agriculture}

Open field agriculture and greenhouses (+Vertical farm) can utilize infrared thermal sensors interconnected to the cloud to detect transpiration cooling of a plant (water stress- fertilising schedules) Hidden sensors can send values of the last temperature (or thermal imaging) together with the intensity of sunlight, humidity level and other meteorological variables, etc. As a result, farm workers can take whatever action is necessary and maximize harvest yield. Sowing, irrigation, harvesting and even soil monitoring has been centralized, also thanks to high precision GPS technology, soil sensors and other systems connected in a batch arrangement. Higher precision of development sensors and actuators could be implemented in the greenhouse. This is an application that was developed in a dizzying way in the last years (with or without LP-WAN), especially with the use of drones in large farms (see 3.2). LP-WAN what means low-power widearea network and might be the most appropriate connection protocol in this case. Probably 5G fixed (router) wireless broadband will be the future solution for rural areas and drones. The improvement of productivity can be enormous. IoT / Al will increase the applications of precision farming techniques which will allow farmers to use and fertilize water, soil and chemicals even more wisely in the future.

\subsubsection{E-commerce - Retail}

In the future, facial recognition (thermal + visible camera) in retail offers could offer interconnected real-time demographic data to improve business strategy. The variation in lighting conditions causes degradation in the performance of face detection; thus, infrared cameras are helpful in this sense. The SWIR (expensive) can also segment human faces much more accurately than the face detection systems of the other visible bands. Theoretical and experimental arguments say that near infrared band is particularly advantageous for detection in human face (disguised faces). Facial recognition is an enormous interconnected tool in all type of security activities.

\subsubsection{Fire Detection - Floods}

Fixed thermal cameras can be used to detect incidents of fire in national forest parks (complemented by drones, section 3.2), in coal pile due to potential ignition, in potentially combustible storage and chemical waste areas, tracking fire detection of deposit assets (heat maps). Coastal erosion and potential flooding areas could be monitored with infrared (+ visible) cameras and integrated in IoT. The images sent to the network could be used to evaluate what is happening or potential risks, and if immediate actions are required. Another example: a robot (with an odometer, that is, an instrument for the measuring distance travelled by a vehicle) duly prepared with a thermal camera can lead access to hard-to-reach areas like a fire, avoiding situations of risk for firefighters or in other situations involving chemical spill, nuclear escape or potential terrorist attack. These potential applications considerably reduce the danger of human inspection / intervention at first. An loT robot with a thermal camera and Al (integrated processor + algorithm) in a fire scenario could face itself basic problems without putting first responders at risk.

\subsubsection{Gas Leak Detection}

This could be a great application in real time with loT and Al (fast + powerful data analytic capabilities+ integration). In fact, it already is and in some cases these systems are today connected to internet by Ethernet (cable). There are several systems for continuous monitoring flare emission (flare stack) and gas leak detection. The systems can visualize leaks and control flares in marine platforms, liquid natural gas terminals, petroleum refineries, chemical plants, landfills, etc. Each system (camera / infrared gas sensors) is sensitive to a different region of the spectrum and usually to a specific band inside SWIR / MWIR / LWIR. Depending on the gas or application, multispectral or hyperspectral cameras can be used. The list of gases that can be detected is very large and this depends, as it was said, on the band. Camera specification (NETD etc.) define the concentration of gas and leak $\mathrm{kg} /$ year possible to detect for certain gas. Some popular gases that can be detected are in the group of methane $(\mathrm{CH} 4)$, propane $(\mathrm{C} 3 \mathrm{H} 8)$, butane $(\mathrm{C} 4 \mathrm{H} 10)$, ethane $(\mathrm{C} 2 \mathrm{H} 6)$. However, they can be monitored in between many others: sulfur hexafluoride (SF6), carbon monoxide (CO), and so on. The goal is to avoid accidents, save money in gas losses and reduce environmental emissions. 


\subsubsection{Geology - Seismological Geodesy}

This has to do with volcanoes (magma = lava and gases), mud volcano (not lava / organic) geysers (thermal source that periodically emits a column of hot water and steam in the air) and other potentially seismic activities. Thermal cameras (+ visible) installed outdoors with adequate FOV, Al and infrared sensors connected to the network and other associated sensors could predict different types of activity and characteristics of volcanoes and, for example, avoid a bright avalanche. Previously, it could characterize if the eruption is effusive or explosive (yellow, orange or red alert) and also the size of the eruption (commonly known as Volcanic Explosivity Index or VEI). The activity in the VEI is the Hawaiian eruption, strombolian eruption, volcanic eruption, plinian eruption, lava domes, and super volcanic eruptions. See also aerial applications in section 3.2 - Aerial (Drones-UAV).

\subsubsection{Home Automation or Domotics}

Internet of Things in homes is the interconnection of several physical objects, including washing machine, toaster, refrigerator, garage, locked doors, microwave oven, coffee maker, HVAC, dehumidifier, thermostat and radiant slab, home entertainment, home theater, blinds, curtains, interior and exterior light of the house, phones and emails, outdoors presence detection, switching off gas supply, etc. All these connected elements can be integrated with sensors, software, electronics and Internet connectivity to exchange information between them and, for example, your mobile phone and they will be able to be managed remotely. For example, a thermal imaging camera with computer vision algorithms can track human movement in a completely dark garage, in a garden, by a fence, and at the main door and even check pet's behaviors. For home security, thermal cameras are an excellent application. For example, in the above cases the thermal cameras with Al can advise people inside houses to block the doors or that if the house is empty, they can automatically call the police. Also, could automatically direct a small fan connected towards a person who feels hot or an infrared sensor can detect heat, for example, on the floor and illuminate the bathroom when a person gets up at night. It seems that our houses are aiming for a 24/7 Internet connection.

\subsubsection{Heath Care}

The presence of elderly people could be detected and controlled online. Body temperature could be measured in care applications for the elderly, and compact thermal imaging cameras could be used to detect activity, presence and falls in critical and dangerous areas, such as bathrooms, preserving privacy (low resolution of the thermal camera). In limited and complex situations, a previously contracted ambulance service that is connected to the network could be automatically notified. Each loT application will use cloud computing and most will enable remote / mobile notifications that can also monitor the medication intake of the elderly. Another application is the robot (humanoid) with sensors (including thermometry) that takes care of the elderly living alone. It could also help the elderly by detecting vital signs.

As medical devices, wearables, electronic medical records (EHR) and other medical information systems become more integrated and are all connected, an evolution that will allow continuous, fully digitized and connected health control (ambulance) will be able to be seen. Today some wearables can measure heart rate, temperature, pressure and movement, as well as body fat and hydration levels. In the same way, wearables can give the location and biometrics of the person who uses it. This brings up issues related to privacy, data ownership, security etc.

\subsubsection{Manufacturing Industry - Process}

Several industries use real-time short-wave, medium-wave or long-wave infrared image (SWIR/MWIR/LWIR) online process-monitoring devices (cameras / sensors) for production and quality control (steel, paper, glass, plastic, automotive, spot welding, recycling plants, etc.). Every day more industries and applications are added to the list and it seems that, with the advent of smart factories (Industry 4.0 - connected to loT with embedded processors), the image processing industry (included the visible) will play an important role in future production, quality control, plant safety, auditing and business processes. Industry 4.0 is a broader concept that not only implies the increase in productivity and plant security but also the modernization of production and process. It goes far beyond that and it will eventually mean better customer service.

For example, with imaging data (see hundreds of thousands, millions of images) accumulated from video infrared (thermal or InGasAs) processing, machine learning (subset of $\mathrm{Al}$ ) can recognize patterns at any specific industrial process and then judge if the process is developing correctly or not. This requires developing algorithms based upon historic data applied for each case. The characteristics must be identified by an expert and then deliver the domain and the type of data (pixel values, textures, contrast, position, shapes, orientation). The capacity of most machine learning algorithms depends on how accurately those characteristics can be identified and extracted. Machine learning $(\mathrm{ML})$ can reduce human errors and more rigorously analyze our increasingly complex imaging (thermal + SWIR + visible) examinations. It is clear that data are the lifeblood of the IoT (Al). It is important that we distinguish the traditional automation in the industry from the IoT smart factory that includes the entire industrial process until the product reaches the end user. The level of automation will depend on each type of industry and on each specific company. 


\subsubsection{Maintenance}

This can be a great application of loT (Industry 4.0.) specifically to important parts of large equipment. Thermographic analysis (heat map) in conjunction with other tests help to diagnose causes. With the aid of loT sensors from several technologies, maintenance managers together with Al will be able to better predict when a breakdown of specific equipment occurs. This is based upon current information and historic records (statistics, big data) from, let's say, infrared thermography, which detects radiance $(L)$ to measure and analyze the heat of any objects, and vibration analysis, which detects wrong vibration signal (potential fault detection) in rotating and reciprocating machineries. Passive ultrasound helps to hear issues not audible to humans. Motor circuit analysis enables analyzing motor health, that is, the detection of electrical imbalances, tribology analysis of friction, wear, lubrication, and particles in fluids that may prove mechanical wear. All the information together and with data analytics (suggest conclusions) allows maintenance to be smarter at generating quick alerts and to make the best decisions. Using the loT(Al), maintenance managers can connect their stockrooms to track orders, incoming shipments or low stock. Maintenance in the loT era means smart factory, less assets shot down, reduction of human error, including industrial accidents, and a loT-based useful information (data) for production and process departments

Portable infrared cameras (call also thermography camera) have been (and are) very useful for many years in maintenance and preservation in industries, facilities, power plants, buildings, infrastructure, etc. In addition, and especially in large plants, fixed infrared cameras / sensors have grown in applications (maintenance process) and will grow even more when it is smart.

As fixed infrared cameras / sensors with IP come down in price, there are several sensitive maintenance applications in which the IoT online connection can be used to replace the work of the portable camera. We would also have to add the application of industrial processes that are being carried out with portable cameras intermittently and that now with the low cost could be done with the fixed loT sensor $24 / 7$

The portable IR camera seems its use will grow as the infrared industry grows and new applications arise, but in part it can be replaced by fixed sensors which give fresh information continuously in an intelligent way. Portable will never stop using and could be a great companion for fixed loT cameras. For example, in the case of special applications, risky scenarios or confirmation of faults (using different optics closer to the target)

Complementing, today there are portable cameras that have built-in Wi-Fi and other connection protocols (BLE) that can be integrated into loT platforms to exchange information, such as images or videos and data.

An alternative that should not be ruled out is the use of an loT robot with visible and infrared cameras to remotely and routinely control critical or risky points for people. The development of a robot of this type will only be possible for some plants (type of enterprise) and also with its infrastructure defined and appropriate for a robot, of course also its costbenefit ratio.

We must don't forget the optical pyrometers. (infrared thermometer) Pyrometers (non- imaging device) were one of the first non-contact infrared technologies. It is very useful, versatile in maintenance and other areas of industry for temperature measurement and it is likely that loT can be integrated.

\subsubsection{Infrared Non-Destructive Testing and Materials Evaluation}

Infrared non-destructive testing (IRNDT) is an effective method for non-destructive testing of several materials. This technique detects not only the smallest defects on surfaces, but also structural defects or air bullets under the surface. Contrary to other inspection methods, thermographic solutions are well-suited for inspections of materials like composite materials: CFRP / GFRP sandwich structures.

It is non-intrusive and non-contact. Depending on the configuration and specimen, it can take from seconds to a few minutes to perform the testing and it is useful to inspect large areas. Maybe this application is one the most difficult to implement in the infrared industry. Therefore, it requires very high qualified people. IRNDT can be carried out either in active mode or in passive mode.

The most popular active tests are performed with optical excitation: pulsed thermography (flash lamps-transient) and lock-in thermography (thermal wave sinusoidal-steady state). There are other types of excitation (vibro, eddy current etc.) and other materials (metals, ceramics etc.) that can be tested. The methodology to be use is determined on a case-bycase basis.

The passive test (the only target of interest is naturally at a higher or lower temperature than the background) does not require excitement; thus, it implies less hardware. Passive is not the most usual test in IRNDT. In most cases, the configuration requires a source of thermal excitation (active thermography). An experimental set-up for active infrared thermography requires, for example, computer-heating control unit—digital imaging acquisition -heating lamps - software (modular architecture normally can change).

Whatever methodology is used, after the test, a raw infrared image is obtained. Then the signal processing is generally applied (data processing algorithm) over the raw to improve the SNR. There are many signal processing techniques such as thermal contrast, differential absolute contrast (DAC), principal component thermography (PCT), thermographic signal reconstruction (TSR), and pulsed phase thermography (PPT). Data processing techniques are required to enhance contrast, to improve the spatial resolution and to increase the signal-to-noise ratio of the infrared signal. 
Imaging evaluation in active thermography (raw or processed) is mostly visual. However, in very repetitive or serial test applications, algorithms, and methods of machine learning (ML) and deep learning (DL) could be developed to make the test more automatic and in real time (Al)

A real example of passive inspection in NDT with AI / IoT (not IRNDT this case) is made by a robot called AirCobot (origin of Airbus). This robot is able to perform visual inspections of the lower parts of an aircraft (fuselage, wings, turbine engines, horizontal stabilizer, vertical stabilizer, tail fuselage, elevators aircraft, undercarriage etc.) in any weather or light conditions. It helps copilots (at the gate) and maintenance personnel perform duties faster, more reliably and with repeatable precision. During aircrafts inspections, the robot makes a thorough diagnosis. This prototype was developed in three domains: autonomous navigation (it moves with its wheels on the gate, around and under the plane), visual non-destructive testing and human-robot collaboration (see upper parts drones in 3.2 Aerial Drones IR sensors). The information collected by the robot under the plane in a pre-established route is sent via loT to the plane cockpit so that the captain and the first officer can make final evaluation before departure (Al / loT only artificial light requires night).

One type of application or similar that could be very interesting to develop IRNDT - IoT artificial intelligence and monitoring is the control of spot welding made by robots in the automotive industry. (e.g.) Each robot with an LWIR uncooled camera can keep track of the work it does every day and turn on the alarms if it is doing it wrong after process in the cloud. (or fog computing /edge computing) The amount of information (big data) that this application develop is enormous from which you can extract welding patterns (machine learning) and classify if there are welding defects or not. The good use of it can lead to have a very efficient automatic quality control.

\subsubsection{People-Animals-Objects / Counting}

This is related to motion / movement detected with IR technology in IoT. Low resolution thermal imaging (microbolometer LWIR $=8 \mu \mathrm{m}-14 \mu \mathrm{m}$ ) is one of the leading methods of counting people in challenging lighting conditions and outdoor settings. People counting and tracking is a relatively new capability to determine how many people are in retail stores, supermarkets and shopping malls and what their habits are while shopping. People counting is also used for managing lines and wait times and making assessments of the total number of people (demographic counter) and their movements in public areas like airports, banking and financial institutions, corporations, educational establishments, entertainment places, government organizations and offices, healthcare centers, retail stores, supermarkets, shopping malls, grocery stores, sports, transportation hubs around the world, among others. Infrared sensors for counting in smart transportation system can be included (3.1.15 Smart Cities).

The main aim of intelligent transport systems is to minimize traffic congestion. Infrared sensors for counting passengers and vehicles are useful for that goal (+ GPS = location; accelerometers = speed; gyroscopes = direction; RFIDs = vehicle identification). Furthermore, infrared sensors would applicable, for example, in a low-light environment to count moving objects that they are naturally at a higher or lower temperature than the background, with a clearly defined infrared signature (Radiosity+ shape and size of the object)

\subsubsection{Security -Surveillance}

Maybe this is one of the areas of greater application of IR in loT. There are already many applications of thermal cameras (+ SWIR) and visible with their IP and they are linked to a monitoring center. The identification of objects in different degrees of illumination and atmospheric transmission (penetration through light fog, dust and smoke) can improve safety by alerting personnel to possible hazards. If we compare MWIR and LWIR thermal bands, LWIR is more efficient when we refer to remove solar glints and more sensitive through smoke and obscurants. There are compact systems that use low-power components, which facilitates the integration of the monitoring system in platform or mast with embedded processor. Then specific algorithms can offer the ability to convert what has been monitored into intelligent video for reliable and fast action (smart cameras: intelligent video systems). The central role of video surveillance continues to be to protect people, private property, industry in general, government offices and infrastructure.

In the case of infrastructures, there are several areas of applications for security with remote infrared IP cameras: transport infrastructure (airports, roads, ports, channels, railways, subways, bridges, buses, etc.); energy infrastructure (dams, wind, thermal, nuclear, high voltage, medium voltage, low voltage, transformation, distribution, public lighting); heat distribution networks (urban heating); infrastructure oil networks (oil pipelines, gas pipelines); drinking water networks (reservoirs, treatment and distribution); drainage networks (sewerage or sanitation and sewage stations); recycling networks (waste collection, landfills, incinerators); infrastructure in telecommunications; hospitals and health centers; education (colleges, institutes, and universities); infrastructure recreation (parks and gardens), etc. There is an endless list of potential applications of remote cameras systems with potential loT real-time connection and skills to understand and interpret situations (AI).

For each application, different types of sensors or cameras can be built, which can be shared with other bands of the spectrum: fixed network IR cameras / PTZ surveillance / dual visible / thermal integrated system (+ SWIR). In each case, for the purposes of choosing the configuration, lenses (very expensive for long range systems) for a given application should be considered using Johnson's Criteria; namely: detection, recognition, and identification (DRI). Detection is the ability to detect that something is there; recognition is the ability to classify the object (for instance, a vehicle is there); and identification is the ability to describe the object in detail (for instance, the vehicle can be a truck).

I cannot fail to mention a very popular security IR sensor that could adapt to the smart grid. This is the passive infrared (PIR) sensor. These sensors (non- imaging) are inexpensive, easy to use and to deploy. PIR (Fresnel lens) detects 
IR-L radiance $\left(\mathrm{W} \cdot\right.$ steradian $^{-1} \cdot \mathrm{m}^{-2}$ ) emitted by moving animals or humans, for example, around fences / perimeter enclosures. They can be an early detection tool and be integrated into the entire IOT security system.

In extremely dangerous situations (indoors / outdoors), another application that can be generalized in the future is the use of robots (IoT), equipped with lights, visible and thermal cameras, for search or rescue operations, or a police patrol.

\subsubsection{Refractories and Insulations}

This is an application that is often done with portable camera. Nevertheless, there are companies that nowadays have specific cameras (with ethernet) not only to control refractories or insulation in applications such as kilns, ovens, ladles, boilers, process heaters dryers, and heat exchangers furnaces, but also to be able to find the hot spot (refractory degradation/ insulation failures) and to protect the person and the assets. In some cases, this is an application of thermal imaging that can be very important, such as following the refractories of molten steel ladles in which a failure could be catastrophic. These are assets that should be monitored on a permanent basis and the study of patterns of images (ML) could lead us to develop application with artificial intelligence bias. Fixed infrared cameras with loT / artificial intelligence could be very useful and proactive in controlling refractories / isolation and thus avoiding unscheduled shutdowns and assets losses. Big building envelope/roofing could be another alternative for the application.

\subsubsection{Smart Cars - Trucks - Agriculture Equipment - Mining Machines - Autonomous Vehicle Construction (haul trucks) - Autonomous Electric Minibus - Watercraft - Robot Patrol Autonomous Vehicle}

High-resolution LiDARS struggle in rain, fog, and snow. Radars can see through bad weather but deliver less detailed information, while visible cameras have the same limitations as human eyes when faced with bright sunlight, glare, fog or nighttime conditions. These sensors limitations can be overcome with thermal cameras or, in some cases, with SWIR cameras. With infrared cameras, there is no problem with the absence of lights at night in farms (harvest agriculture equipment) or poorly lit harsh environments like mine or its surroundings with smog, smoke, fog or rain. In general, the autonomous vehicle could have a set of those sensors connected to loT to observe the environment and will autonomously make decisions about its behavior and navigate with a high level of security or pass the information to a human operator at a different location who will control the vehicle through teleoperation.

Farming, construction and mining vehicles (plus mining machinery) can be dangerous to work due to the inherent risks of handling large equipment and other environmental factors, like hazardous work sites. Unmanned machines can operate in a field to accomplish tasks efficiently and in a much safer way than humans do. Reducing latency is basic (LTE $5 G$ - reduces response) to avoid obstacles, pedestrians, animals, another vehicle, etc. Another loT application in relation to cars is the use of thermal low-resolution sensors connected to mobile phone for detecting living beings.

For instance, it could be used to prevent children or animals from being left inside a car which is too hot (in summer) or too cold (in winter). Autonomous vehicles (smart mobility) in general pose a question: What will happen in terms of insurance coverage? In other words, who will be responsible for traffic accidents if this activity is carried out by a car and not by human beings?

\subsubsection{Smart Cities}

Infrastructures lights could be dimmed in some places of cities or towns to save energy and can be switched on when thermal sensors detect motion (PIR o similar). Advertising posters could shine only when a passerby walks the area. Thermal sensors with IP connected to loT network would imply a decline in energy cost by equipping street lights in cities with sensors connected to a transmission network. Intelligent street lights that can see, hear, and perceive their surroundings are generating data that empowers urban services.

The challenge is to achieve greater energy efficiency allowing the lighting of buildings and the exterior to adjust according to the presence of people, vehicles, and also allowing an optimized acclimatization depending on the season and local climatic conditions (totally city integrated, intelligent and sustainable). The door will know when someone leaves the office. Traffic will be able to be avoided by smart parking, that is, by consulting the server with the GPS location of a vehicle, the smart system will take people to the nearest parking place available. Other applications could be smart waste management, environmental monitoring, smart traffic management and water disaster management. Intelligent Transport System (ITS) application which monitors the traffic in a city is consider part of IoT (Toll collection, automatic surveillance of traffic violations, emergency notification system on board the vehicle, automatic speed control, control congestion rates, other)

Pedestrian safety awareness could be enhanced at crosswalks via networked LiDAR, visible, thermal imaging, and other sensors, which could detect pedestrians' position, height and width for security and statistical purposes. Tracking moving objects could be used to detect traffic patterns, leading to better planning of infrastructure in cities.

With respect to water supply, a centralized water planning strategy with different sensors (connected loT) could be used in storm drains, water storage tanks and water supply lines. Water requirements in terms of domestic and industrial use and for activities such as gardening and retail could be estimated. Smart gardens in cities can be very useful in watering plants and flowers in the right places at the right time. Thermal images (+ other sensors) can be used in this application.

When intense rains occur depending on the city and the area floods can occur. The difference in heat capacities between the soil or asphalt (background) against the water shows normally an define infrared signature that could be 
detected by thermal devices. This infrared device connected to the a loT cloud and in turn the cloud is connected to other types of sensors can bring a quick response to the emergency.

\subsection{Aerial Drones - UAV}

Table 1 (page 13) shows a list of potential applications in alphabetical order for infrared industry - loT on aerial devices (drones). No doubt that thermal imaging devices and loT will fuel large-scale growth of drones. IoT and drones are two contemporary, relatively new technological areas belonging to the same generation and they are both in their immature stage. UAV / drones require special chapters in loT. Someone called this the Internet of Drones (loD).

We will not mention in this paper nor will we detail in table 1 (below - page 13) submarine drones. The reason is because the water is opaque for infrared and because wireless communications such as Wi-Fi and other standard radio signals do not travel well through the good electrical conductors such as salt water (seas).

Any way for many other applications there are international efforts to develop technology to connect to the Internet the gigantic water mirror seas-lakes-rivers and their depths (73\% of the surface of the Earth) Potential applications: ecological monitoring. ocean acidification, water quality/ salinity and pollution, tracking fish, navigation assistance, earthquake and tsunami warning, surfaces \& underwater eye of a hurricane, shipwrecks, pressure, and temperature of water, sea-lakes-river levels, surveillance etc. That's call it the Internet of underwater objects (loUT).

Drone in all its uses and applications are still in its infancy, but they are growing very fast. There are many advantageous applications which could involve drones and the infrared devices-loT (+ visible devices). If they are handled efficiently, they can save lives and a lot of money. The future is unthinkable, with no limits. However, to date air traffic and radio transmission management still have regulatory limitations especially for private properties, airports, harbours, towns and cities.

Normally commercial drones can be piloted in two different ways: either line of sight by visually observing the drone or by first person view (FPV). In an FPV system, the video image from an onboard camera could be transmitted by radio to a personal video display on the ground in the form of a screen or video goggles. As it has already been said, wireless video for FPV drone piloting is immature technology. FPV is highly regulated or it is directly prohibited.

It would be necessary to harmonize drone's regulations and potential certification standards and procedures internationally, not only the rules of use of the airspace and radio space (Jamming and range) but also the integrated safety features of drones themselves (e.g. obstacles avoidance: downward, front, rear and upper). Drones-UAV are very advantageous, but non-professional use of them could be risky and dangerous. Drones operators must be very well trained and licensed.

Equipped with thermal images (+ visible cameras), InGaAs, UV or multispectral cameras, microphones, hyperspectral or LiDAR sensors and others, drones have countless applications and a fascinating future. Experts say a drone can collect more dramatic or accurate pictures than a helicopter (things at rest and things in motion).

It would be very helpful to expand the use of drones with thermal sensors as essential tools for emergency preparedness and post-incident missions. The autonomy (battery) and weather conditions to fly are always concerns or limitations.

Wireless could be by LP-WAN---WAN IoT. Another way drones can send a video or picture is to send to a transmitter that encodes the video into a radio waves and then a receiver that is set to same frequency receives the signal and converts it back into video.

It is thinkable for us to imagine, in the future, a drone with artificial intelligence that, in a certain way, can make its own basics decisions (like an aerial robot or smart drone).

\section{Conclusion}

Inside this paper we have made a general review and some futurology of challenges and risks of loT as a network of physical devices and an approach of involvement between this network and the infrared industry. (thermal imaging point sensors etc.) It is a brief review of a very broad topic that grows day by day. We are at the dawn of a new era with vast future. The infrared industry in general, loT, artificial intelligence, drones, all these technologies or disciplines with different origins are producing amazing results and grow exponentially each year.

In the universe of infrared applications, we must bear in mind that them could be carried out as qualitative quantitative / passive -active / radiometric - non-radiometric. Furthermore, applications could be put into practice in different bands of the spectrum SWIR-MWIR-LWIR / Hyperspectral - Multispectral / Fusion image: IR-Visible. Each case must be analyzed to evaluate which band is suitable for the application

The most important final product of the Infrared industry devices are basically images (thermogram) and infrared videos (machine vision- computer vision), both visible to the human eye and representative of the infrared radiation (invisible to humans) that emits or reflects the object or objects that we are observing. When we talk about images, depending in application we can find a lot of information in each of them. When we have many images (+data) of the same application they can be classified and analyzed to find patterns (data analytics) That way we're on our way to loT, artificial intelligence, supported by machine learning and deep learning. 
Table 1. UAV—DRONES IoT Aerial systems --Potential IR-UAV applications (1)

\begin{tabular}{|c|c|}
\hline Fields (2) & Some areas of applications (+IR-visible - IoT) \\
\hline Agriculture & $\begin{array}{l}\text { Cattle count - Greenhouse -Precision agriculture -Pest and disease } \\
\text { control mapping plant growth moisture levels, yields. Visible reflectance } \\
\text { maps of crops-thermal emission }\end{array}$ \\
\hline Aircraft Inspection & $\begin{array}{l}\text { Drones can inspect upper parts of the aircraft. Using visible cameras, and } \\
\text { the possibility of passive thermographic techniques. The algorithms for } \\
\text { automatic detection of defects, with a machine learning software, can } \\
\text { identify various elements (defects) on fuselage: texture irregularities, } \\
\text { cracks in pitot probes, rivets, openings, text, defects, corrosion, oil stains, } \\
\text { lightning strikes, hail stone damage, fuselage regulatory stickers. }\end{array}$ \\
\hline $\begin{array}{l}\text { Cargo Delivery -- product deliveries (3) Air Taxi } \\
\text { efficient delivery to a remote place }\end{array}$ & $\begin{array}{l}\text { Unmanned - Food -retail -e commerce-- A higher payload inevitably } \\
\text { requires longer battery life and larger vehicles -use for parcel deliveries }\end{array}$ \\
\hline $\begin{array}{l}\text { Construction - Infrastructure Inspection - Road condition } \\
\text { control }\end{array}$ & Inspecting bridges - Roads \\
\hline Drone fly and drive & $\begin{array}{l}\text { New prototype for a system of wheeled, autonomous drones that can } \\
\text { switch between flying and driving (multifaceted loT application) }\end{array}$ \\
\hline $\begin{array}{l}\text { Emergencies \& Disasters. } \\
\text { recovery strategy }\end{array}$ & $\begin{array}{l}\text { SAR - First Aid-relief during natural disasters - Flooding--Medicines for } \\
\text { a critical patient waterproof payload. Post-incident missions. An urgent } \\
\text { spare part to a shut-down oil rig. Ambulance drone with a defibrillator. } \\
\text { Lifeguards used drone to drop an inflatable life preserver in just minutes, } \\
\text { Earthquake: miniaturized drone with a thermal camera to find trapped } \\
\text { people. - Hurricanes - Tsunami Warning }\end{array}$ \\
\hline Environmental \& Wildlife Conservation & $\begin{array}{l}\text { Oil Spill -- Beach Sharks monitoring-- Wild animals in coastal waters and } \\
\text { open land- Surveillance of sea mammals-- Detection of the spread of } \\
\text { algae }\end{array}$ \\
\hline Fire fighting & $\begin{array}{l}\text { Houses -Buildings-Ships-National Parks - Brushes fires Neighbourhood } \\
\text {-Open land-- Early Fire Detection. Drone which can send the image of } \\
\text { scene back. Find source of a fire. }\end{array}$ \\
\hline Gas Leaks \& Chemical leak -Gas Flare & Infrared Inspection flare stacks and chimneys \\
\hline Insurance industry & Claims attention: visit accident sites with drones to collect data \\
\hline Media \& Communications & $\begin{array}{l}\text { Films-Journalist-Marketing-Advertising-Photography-Television- } \\
\text { Broadcasting - Thermal }\end{array}$ \\
\hline Mining & $\begin{array}{l}\text { Intelligent drilling equipment and. Predictive maintenance in biggest } \\
\text { shovel and other mining equipment } \\
\text { Coal mine fire prevent Infrared thermography technique with GPS }\end{array}$ \\
\hline Power Lines - Wind Turbine I - Pipe Lines & $\begin{array}{l}\text { Overheating nodes - Detecting leaks - Power Grid-Structural. Visible - } \\
\text { Thermal-- Drones, for example, are being used to monitor remote } \\
\text { pipelines }\end{array}$ \\
\hline Roofing - Flat Roofs- Sloped Roofs - & $\begin{array}{l}\text { Missing or broken shingles- Masses of moss- Moisture intrusion - } \\
\text { Missing insulation - Leak - Inspection construction defects }\end{array}$ \\
\hline Scientific & Glacier surveillance-- Iceberg monitoring- Biodiversity \\
\hline Security - Public safety & Crime Scene - Security and Surveillance - Unrest - Coastal security \\
\hline Smuggling & Borders - surveillance and monitoring purposes \\
\hline Solar Panel Inspection & $\begin{array}{l}\text { Infrared signatures show defective panels -- detect hot spots before they } \\
\text { become breakdowns. }\end{array}$ \\
\hline Telecommunications -Cell towers & Inspection = visually - thermal \\
\hline $\begin{array}{l}\text { Topography Geoscience-Seismic and Volcano Activity } \\
\text { (3.1.5) Hilly Terrain }\end{array}$ & Detailed mapping or charting - Volcanic eruptions - surveillance \\
\hline Traffic Safety Control & Car accidents \\
\hline Train tracks - Railway safety & Train line inspections - water - sleepers \\
\hline 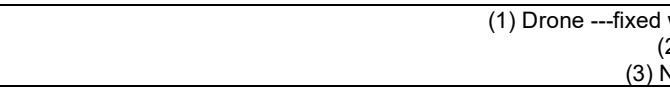 & $\begin{array}{l}\text { g planes - multi-rotors -single rotors } \\
\text { ר alphabetical order } \\
\text {-commercial reality yet }\end{array}$ \\
\hline
\end{tabular}


The final success of the loT will depend on the challenges to be overcome. Some are general, and others will depend on each industry and application. Basically, the keys are the miniaturization of cameras / sensors, the progress of artificial intelligence (for an loT with more added value), the investments in networks and the levels of cybersecurity that can be achieved in each project or system. Another key to success in any of the potential loT / Infrared applications is choosing the right platform and its layers (connectivity, hardware, software, analytics, user interface, security...) that fits integrally or partially into our business and can be improved and updated over time.

loT as an overall technology will help human beings not to forget things, to avoid accidents, and to be more orderly, efficient, and productive, but, in some cases, it could generate catastrophic situations. More data means more accurate results and predictions. The key lies in each situation and application. Each end user must establish where they want to go, their cost - benefit of what they want to perform.

On the one hand, intelligent things could be more precise and consistent than humans in communication starting analysis from a large database. (+Al) On the other hand, as people grow, they usually learn, develop and use hundreds of different skills. In extreme or confusing situations, seems human intelligence is more versatile and secure than artificial intelligence. However, things-machines (infrared devices) with artificial intelligence can carry out specific operations (Al) more accurate and much faster than humans as many times as necessary without fatigue and with much memory capacity. Moreover, computers, cameras, and sensors do not need vacation or rest; they can work in an uninterrupted way. It seems as if the world is moving in all fields and disciplines towards digital integration.

IoT is on its way to be a network connected to a gigantic sea of sensors that correspond to things / objects that are part of our planet and the infrared industry itself will be a potential contributor to that network with very specific detectors-sensors.

\section{Acronym List}

- Al: Artificial Intelligence

- $\quad$ AGI: Artificial General Intelligence

- API: Application Programming Interface

- CFRP: Carbon Fiber Reinforced Plastic

- DCS: Distributed control systems

- DL: Deep Learning

- Drone: Male Bee

- FPA: Focal plane array

- Gateway: Two networks/ different protocols-translate one into the other.

- GFRP: Glass Fiber Reinforced Plastic

- GPRS General Packet Radio Service

- HOT: High Operation Temperature (detectors)

- IARC: International Agency for Research on Cancer

- $\quad$ IloT: Industrial Internet of Things - Industrial loT market

- IoT: Internet of Things -- Consumer market

- IP: Internet Protocol address

- IP4V: Internet Protocol Version 4

- IPv5: Internet Protocol Version 5

- IP6V: Internet Protocol Version 6

- I: Radiance intensity $\left(\mathrm{W} \cdot\right.$ steradian $\left.^{-1}\right)$

- InGaAs: Indium gallium arsenide (SWIR)

- IR: Infrared

- ISP: Internet Service Provider

- L: $\quad$ Radiance $\left(\mathrm{W} \cdot\right.$ steradian $\left.^{-1} \cdot \mathrm{m}^{-2}\right)$

- LAN Local Area Network (Wi-Fi + thernet + Fiber optics)

- LiDAR: Light Detection and Ranging

- $\quad$ LP-WAN Low Power Wide Area Network

- LTE 5G: Long Term Evolution - Fifth generation of wireless technologies

- LWIR: Long-wave infrared

- MAC : Media access control address (MAC address)

- M Radiant exitance $\left(\mathrm{W} \cdot \mathrm{m}^{-2}\right)$

- ML: Machine Learning

- M2M: Machine to Machine

- MQTT: Message Queuing Telemetry Transport

- MWIR: Mid-wave Infrared

- NAT Network Address Translation

- NETD: Noise equivalent temperature difference

- NFC: Near field communication

- NI: Natural Intelligence

- PAN: Personal area network (Bluetooth + ZigBee + wired USB) 10 meters,

- PIR: Passive Infra-Red sensor

- PTZ: Pan-Tilt-Zoom

- RFDI: Radio Frequency Identification

- SAR: Search and Rescue 
- $\quad$ SCADA: Supervisory Control and Data Acquisition

- SNR: Signal-to-noise ratio

- SWaP: Size, Weight and Power

- SWIR: Shortwave Infrared

- T: Thermodynamic Temperature

- TCP/IP: Transmission Control Protocol/Internet Protocol

- UAV: Unmanned Aerial Vehicle

- WAN: Wide Area Network

- $\quad \varepsilon$ : $\quad$ Emissivity - (material's ability to emit) - dimensionless quantity

- $\quad \rho: \quad$ Reflectivity -- dimensionless quantity

- $\mu \mathrm{m}$ : $\operatorname{micron}\left(10^{-6} \mathrm{~m}\right)$

\section{REFERENCES}

[1] Steven W. Brown, Bettye C. Johnson, Keith R. Lykke NIST Radiometry: Sensing the World Published: February 01, 2001 (1)

[2] D.L. Balageas, J.-M. Roche, F.-H. Leroy Comparison and ranking procedure for an objective assessment of thermographic NDE methods

[3] Sandra Dominikus and J"orn-Marc Schmidt IAIK, Connecting Passive RFID Tags to the Internet of Things Graz University of Technology Graz University of Technology

[4] Domotics and Robotics Dario Russo Institute of Science and Technologies of Information (ISTI) Domotics Lab

[5] Internet of Drones Mirmojtaba Gharibi, Raouf Boutaba, Fellow, IEEE, and Steven L. Waslander, Member, IEEE

[6] Development of an 'Internet of Things' (IOT) based thermography inspection system PhD - Cranfield Doctoral UK

[7] Toward Wearable Wireless Thermometers for Internal Body Temperature Measurements Zoya Popovic, Parisa Momenroodaki, and Robert Scheeler

[8] Drone Analyst--Why Drones Are the Future of the Internet of Things -Colin Snow

[9] Internet of Things: Architectures, Protocols, and Applications Pallavi Sethi and Smruti R. Sarangi

[10] An embedded real-time pedestrian detection system using an infrared camera - Robert Walczyk; Alistair Armitag T. David Binnie

[11] Outline of artificial intelligence - Wikipedia

[12] Artificial Intelligence in Non-Destructive Testing Conference: 2014 IEEE 11th Far East NDT/EAt: Chengdu Ryszard Sikora Tomasz Chady

[13] Wolfgang Bisle -Kühlkenstrasse 22 -28717 Bremen Germany

[14] Microsoft FarmBeats: Al \& loT for Agriculture

[15] Internet of things -- Wikipedia,

[16] Enhanced image processing for infrared non-destructive testing F. LopezEmail authorX. Maldague. IbarraCastanedo

[17] Inside the Internet of Things (IoT) Deloitte

[18\} At\&T -- loT

[19\} Forbes notes in artificial intelligence -cognitive computing

[20] Machine Learning Arthur Samuel way back in 1959

[21] IEEE 802 LAN/MAN Standards Committee

[22] IEEE 802-11 wireless computer networking standards working group of the Institute

[23] IEEE 802-15 working group of the Institute - wireless personal area network (WPAN)

[24] IEEE 802.15.1: WPAN / Bluetooth

[25] CISCO Securing the Internet of Things

[26] DJI Drones

[27] Infrared Thermography and NDT: 2050 Horizon by F. Khodayar*, S. Sojasi* and X. Maldague*

[28] What is next for loT \& IloT? Ahmed Banafa San Jose State University | SJSU · Department of General Engineering

[29] IBM IPv4 and IPv6 address formats - Connecting all the things in the Internet of Things

[30] Google What are IPv4 and IPv6?

[31] INTEL Smart Video -Intel Smart Farming

[32] ARTIFICIAL INTELLIGENCE MEET INTERNET OF THINGS W. Malini Prema Kumari Research Scholar,Periyar University,Salem,INDIA 